\title{
USABLE SECURITY AND E-BANKING: EASE OF USE VIS-À-VIS SECURITY
}

\author{
Morten Hertzum, Niels Jørgensen, Mie Nørgaard \\ Roskilde University, Denmark \\ Email: \{mhz,nielsj,mnl\}@ruc.dk
}

\begin{abstract}
Electronic banking must be secure and easy to use. An evaluation of six Danish web-based electronic banking systems indicates that the systems have serious weaknesses with respect to ease of use. Our analysis of the weaknesses suggests that security requirements are among their causes and that the weaknesses may in turn cause decreased security. We view the conflict between ease of use and security in the context of usable security, a concept that is intended to match security principles and demands against user knowledge and motivation. Automation, instruction, and understanding can be identified as different approaches to usable security. Instruction is the main approach of the systems evaluated; automation relieves the user from involvement in security, as far as possible; and understanding goes beyond step-by-step instructions, to enable users to act competently and safely in situations that transcend preconceived instructions. We discuss the pros and cons of automation and understanding as alternative approaches to the design of web-based e-banking systems.
\end{abstract}

\section{Keywords}

Usable security, ease of use, security, electronic banking, public key infrastructure, strong passwords.

\section{INTRODUCTION}

E-banking, which we define as web-based electronic banking, is a high-risk area with a potential for substantial economic loss. The high risk makes security a prime concern. In 2001, an estimated total of $\$ 17$ billion was spent on information-security products and services in the United States alone (IDC, 2003). The diversity of e-banking users and the absence of any special training prior to becoming one make ease of use a prime concern as well.

The current web-based variant of electronic banking is the latest of several generations of systems: Automated teller machines (ATMs) were the first well-known machines to provide electronic access to customers of retail banks. Next came phone banking where users call their bank's computer system on their ordinary phone and use the phone keypad to perform banking transactions. $P C$ banking superseded phone banking and allowed users to interact with their bank by means of a computer with a dial-up modem connection to the phone network. Phone and PC banking entailed maintenance costs associated with keeping up to date with diverse modems and with avoiding prohibitively complex installation procedures. E-banking uses the web browser for the user interface and the Internet for data transfer and download of software, and so has a potential for reducing maintenance costs. For users, e-banking provides current information, 24-hours-a-day access to banking services - in addition to the familiar browser interface. The primary services provided by ebanks are transferring money among one's own accounts, paying bills, and checking account balances. Loans, brokering, share trading, service bundling, and a host of other financial services are being added to these primary services (e.g., Dewan \& Seidmann, 2001). Since the late 1990s ebanking has developed from virtual insignificance to tens of millions of users worldwide, and due to their high penetration, e-banking systems must accommodate a wide range of users. E-banking is widely used in, among other places, the Nordic countries. In 2001, e-banking was used by more than $25 \%$ of the population in Norway, Sweden, and Finland, and by $15 \%$ of the population in Denmark (OECD, 2001). In 2004, e-banking usage in Denmark had grown to 45\% (Statistics Denmark, 2004). The purpose of this paper is twofold. At the concrete level, we assess the security and ease of use of contemporary Danish e-banking systems. At the conceptual level, the study is about usable security - 
that is, matching security principles and demands against user knowledge and motivation. To address these issues, emphasis is on use-related aspects of security, such as risk awareness, installation procedures, and whether systems are oriented toward instruction or understanding rather than on purely technical issues, such as encryption algorithms.

While we discuss usable security in the context of e-banking, it should be noted that similar security issues confront users and developers of e-commerce and e-government systems. Within egovernment examples include the current endeavours toward further digitalization of public administration by providing citizens with access to their medical or tax data via the Internet.

In the remainder of this paper, we relate usable security to usability in general, describe our evaluation method, and present and discuss the results.

\section{USABLE SECURITY}

Passwords provide an illustrative example of the conflict between security and ease of use and, thereby, of the need for a concept of usable security (e.g., Adams et al., 1997; Klein, 1990; Morris \& Thompson, 1979; Pinkas \& Sander, 2002; Schultz et al., 2001). While passwords such as person names or other real words are relatively easy for a user to remember they are weak from a security point of view because they are vulnerable to dictionary attacks. Strong passwords (e.g., x $7 \mathrm{~h} ! \mathrm{t} \% \mathrm{C} 9$ ) are less vulnerable to attack but at the same time more difficult to remember.

The term ease of use is used in this paper in the sense of learnability and understandability of user interfaces, and both ease of use and security are considered to be contained within the concept of usability. Within human-computer interaction, the ISO 9241-11 (1998) definition of usability has gained widespread acceptance. The standard defines usability as the "extent to which a product can be used by specified users to achieve specified goals with effectiveness, efficiency and satisfaction in a specified context of use." This broad definition equates usability with the quality of a system in use and, thereby, addresses the entire issue of whether the system meets the needs of actual users. Specifically, effectiveness includes whether a system enables users to achieve their goals at an appropriate level of security, and likewise, ease of use is also contained within the ISO 9241-11 definition of usability. In contrast, several other studies allude to a conflict between usability and security (e.g., Adams et al., 1997; Dourish \& Redmiles, 2002; Schultz et al., 2001; Smetters \& Grinter, 2002); these studies assume a narrower definition of usability, as conventional in software engineering (see ISO/IEC 9126-1, 2001).

Although security has become an aspect of virtually any system, and users habitually interact with access-control mechanisms for, among other things, personal computers, email accounts, and ecommerce or e-government sites, the security facilities of such systems are often less than fully usable. Contributions to a better understanding of usable security include the set of characteristics proposed by Whitten and Tygar (1999) of the usability problem for security, including the weakest link property, the unmotivated user property, and the barn door property. The latter is the property that once a secret such as a private key has been compromised, then closing the barn door, e.g. setting up a firewall, does not restore security. Several studies including Schultz et al. (2001) suggest that security measures that are inconvenient for users may weaken security, for example because of lack of user acceptance or outright resistance. Dourish and Redmiles (2002) propose a distinction between theoretical and effective security. Theoretical security concerns the level of security that is technically possible; for example, digital signatures provide strong authentication under the assumption that various computational problems related to prime numbers will not be solved within some time frame. Effective security concerns the level of security achieved in practice, and is typically lower than theoretical security, due to weaknesses with respect to, among other things, algorithm implementations, protocol design - and ease of use. Further, a concept of perceived security may be useful to capture the user's subjective perception of the security risks involved in ebanking. Several studies including Jih et al. (2005) indicate that user adoption of e-banking is 
affected by perceived security. This supports a view of security as crucial to the overall usability of e-banking systems.

Whitten and Tygar (1999) suggest the following definition, against which we measure the e-banking systems evaluated in this study: Security-related software is usable if the people who are expected to use it -

1. are reliably made aware of the security tasks they need to perform;

2.are able to figure out how to successfully perform those tasks;

3.don't make dangerous errors; and

4.are sufficiently comfortable with the interface to continue using it.

This definition addresses the effectiveness (issues 1 and 3), efficiency (issues 1 through 3), and satisfaction (issue 4) of security-related software, and so accords with ISO 9241-11 (1998).

There are two ways in which it seems appropriate to re-interpret Whitten and Tygar's notion of a security task for use with our evaluation. First, many security tasks in e-banking are tightly integrated into proper e-banking tasks, e.g. typing a password to confirm a transaction, rather than independent tasks such as in Whitten and Tygar's evaluation of PGP, a tool for secure email. Second, we stress that some security tasks in e-banking may be difficult for the system designers to predict in advance, which seems to contradict an underlying assumption of issue 1 . Thus, security tasks may be part of other tasks, and may not be predicable in advance.

\section{WHAT WE EVALUATED}

A single e-banking service, transfer of money to an account held by someone else, was selected to represent ordinary retail banking, that is, non-commercial account holders' ordinary interactions with their e-bank. We believe this service is the most important for most ordinary users, and certainly every e-bank should provide it in a secure and easy-to-use way.

A sample of six Danish e-banks was selected for the evaluation. The sample includes the three ebanks offered by the three largest Danish banks; these develop their e-banking software in-house. Each of the three other e-banks is based on software from one of three independent Danish manufacturers of generic software for e-banking; all other Danish e-banks are based on software from these three manufacturers, but customization and extension with new features may result in user interfaces that are different from those in the sample.

The task of conducting a money transfer in the six e-banks requires that two or three other tasks are carried out, namely installation (five of the e-banks) and logon and logoff (all six e-banks). We include these prerequisite tasks in the evaluation.

The prerequisite tasks serve to attain various security requirements: All six e-banks have pledged to comply with the security requirements laid down in the industry code defined by the Danish Bankers Association. The code contains the following requirements, rephrased here to comply with standard security terminology (see ITU 1991): User authentication to verify the identity of the user prior to completing transactions and prior to getting access to account information. Nonrepudiation to enable banks to prove that a given transaction has been conducted by a given user. Data confidentiality to protect transaction or account data communicated between the user and the bank from disclosure. There are various more specific requirements, most notably that user authentication is based on two independent secrets:

1.a secret the user knows (e.g., a memorized password)

2.a secret the user possesses (e.g., a private key stored on the user's computer).

The installation task is a prerequisite in the five banks that implement the industry code's two-secret requirement by means of a public key infrastructure (PKI) solution. In contrast, no installation task is required to conduct money transfers or other services in the e-bank that makes use of a physical code card. Table 1 summarizes how the two solutions implement the industry code's two-secret requirements.

AJIS Special Issue

December 2004 
The logon and logoff tasks establish and terminate a secure connection to the e-bank using the SSL/TLS (secure socket) protocol, for data confidentiality. Logon in all six e-banks requires both the possessed and known secrets.

The full evaluation comprises the following four tasks having various specified security subtasks: Installation, with two security subtasks: password definition and signature-file management.

Logon, with one security subtask: user authentication.

Money transfer, with one security subtask: user authentication.

Logoff, which is in itself a security task.

The goal of the evaluation of these tasks is to answer the following questions, which are based on Whitten and Tygar's (1999) definition of usable security:

Is the user made reliably aware of, and able to successfully perform the security subtasks?

Is the user prevented from making dangerous errors?

What is the cost of the security subtasks in terms of added user-interface complexity - is there a risk that the user does not feel sufficiently comfortable to continue using it?

\section{HOW WE EVALUATED}

The e-banking systems were evaluated by a walkthrough of the four tasks (i.e., by analytic inspection). Accounts were opened in the six banks so that we could conduct the four tasks as ordinary customers. The walkthroughs consisted of constructing sequence models of the actions involved in performing the four tasks. The sequence models, inspired by Beyer and Holtzblatt (1998), document the information provided by the systems and delineate the correct sequence of user actions required to perform the tasks. In a separate test we also attempted to define weak passwords, deliberately disregarding any advice given by the systems.

To attain an approximate, quantitative measure of the complexity of the user interfaces, we counted the number of steps, codes, and concepts involved in each task, as recorded in the sequence models. A step is an action users must perform to provide input to the system, either by filling in a field or clicking a link. A code is a character string users must key in to identify themselves, including account numbers and passwords. Concepts are security concepts presented to users as part of onscreen explanatory text.

The evaluation was conducted on a PC with Microsoft's Windows 2000 operating system and Internet Explorer 6.0 browser. The security settings of the browser were set to "custom" and the privacy level to "medium".

\section{RESULTS}

In all six e-banks, the user authentication subtasks of logon and money transfer are tightly integrated. The user merely has to type the relevant passwords etc. when prompted. Serious errors are probably prevented during these tasks, because the tasks cannot be completed until the user provides the input. 


\begin{tabular}{|l|l|l}
\hline & \multicolumn{1}{|c}{ Public key infrastructure (PKI) } & Code card \\
\hline Possessed secret & $\begin{array}{l}\text { The private key. The key is stored in a signature file, } \\
\text { typically on the user's computer, and is stored in } \\
\text { encrypted form, to protect it from disclosure in case of } \\
\text { theft of the signature file. }\end{array}$ & $\begin{array}{l}\text { The physical code card. The card is the size of a credit card } \\
\text { and contains 80 pairs of keys and codes. It has no electronic } \\
\text { or magnetic components; all the information it contains is } \\
\text { conveyed in print. When prompted the user must type the } \\
\text { code that corresponds to a given key. }\end{array}$ \\
\hline Known secret & A password used to decrypt the private key. & A password which is unrelated to the code card. \\
\hline Installation \\
required? & $\begin{array}{l}\text { Yes. Generation by software on the user's computer of a } \\
\text { pair of private and public keys, and storage of them in the } \\
\text { signature file. }\end{array}$ & $\begin{array}{l}\text { No. When the user receives a code card it is ready for use. } \\
\text { Each key-code pair is used only once, in a random order } \\
\text { chosen by the e-bank system. Eventually a new code card is } \\
\text { sent to the user. }\end{array}$ \\
\hline
\end{tabular}

Table 1. User authentication by PKI vs. code card, and how they implement the two-secret requirement of the Danish Bankers Association's industry code.

This integration facilitates daily use of the e-banks. Still, the e-banks suffer from three main weaknesses:

1.The installation task associated with the five PKI-based e-banks is highly demanding, and more complex than any other task. In contrast, the code-card solution relieves the user from installation, but as a consequence each logon is more cumbersome (see Table 2).

2.Security alerts and other messages from the browser are likely to confuse the user during installation (if any) and also during logon and logoff.

3. The user may not be made sufficiently aware of the security subtasks of installation; the user is not likely to be able to figure out how to perform them; and the creation of a weak password is not consistently prevented.

In the remainder of this section we detail observations 1-3. The full evaluation results, including the sequence models, can be seen in Hertzum et al. (2004).

\begin{tabular}{|c|c|c|c|c|c|c|}
\hline & $\begin{array}{c}\text { Ebank1 } \\
\text { (code card) }\end{array}$ & $\begin{array}{c}\text { Ebank2 } \\
\text { (PKI) }\end{array}$ & $\begin{array}{c}\text { Ebank3 } \\
\text { (PKI) }\end{array}$ & $\begin{array}{c}\text { Ebank4 } \\
\text { (PKI) }\end{array}$ & $\begin{array}{c}\text { Ebank5 } \\
\text { (PKI) }\end{array}$ & $\begin{array}{c}\text { Ebank6 } \\
\text { (PKI) }\end{array}$ \\
\hline $\begin{array}{c}\text { Installatio } \\
\text { n }\end{array}$ & $\begin{array}{c}\text { No } \\
\text { installation }\end{array}$ & 13 & 14 & 19 & 15 & 23 \\
\hline Logon & 9 & 5 & 5 & 6 & 6 & 7 \\
\hline Transfer & $3+$ data & $5+$ data & $5+$ data & $6+$ data & $6+$ data & $6+$ data \\
\hline Logoff & 1 & 2 & 2 & 1 & 2 & 2 \\
\hline
\end{tabular}

Table 2. Approximation of the complexity of the four steps in the evaluation by the number of steps they require. The transfer task additionally requires typing in data to specify the transfer, such as the amount to be transferred.

\section{The complexity of the installation task in the PKI-based e-banks}

The complexity of installation in the six e-banking systems is described in more detail in Table 3 based on quantitative data about the number of steps, codes, and concepts involved in the installation process (see the previous section for a definition of these terms). 
A significant part of the installation process is the typing of various passwords and other codes, for identification and authentication. The user must type a temporary identification and a temporary password, for initial identification and authentication, and a permanent identification and a permanent password for subsequent use. All five PKI-based e-banks require users to define their own permanent passwords. Some of the banks merge the two identification codes, reducing the number of codes from four to three, as can be seen in the table. In addition to the codes users are also asked to provide other security-related input, such as where to store the signature file.

The security concepts presented to the user during installation include concepts such as authenticity, verification, signing, certificate, secure connection, private key, and signature file. These concepts are foreign to most users, intricately related, and not easily understood. Users are presented with 814 such concepts during installation of their e-banking system (Table 3). This includes concepts appearing in browser messages (see below). Of course, the number of steps increases beyond those in the table if the user chooses to inspect some of the links to explanatory information or has to backtrack to make changes or correct mistakes.

The complexity of the installation task is aggravated by scant information about how far the user has progressed toward completion of the installation.

The code card-based e-bank avoids installation altogether. Assessment of the merits of the code-card technology against a public key infrastructure must consider the trade-off between avoiding installation and introducing some inconvenience in daily operation. Installation may be a barrier that precludes further use of the e-banks, but is performed only once. The three other tasks will be executed repeatedly during everyday use of the e-banks. The code card is an external device and everyday use is somewhat less convenient because the card must be available. Also, logon to the code card-based bank involves more steps (nine) than in the other e-banks, and the user is presented with 11 security concepts during logon. For logon and money transfer the user must type four codes, including the code-card number and a key, both of which must be read from the card.

\begin{tabular}{|l|c|c|c|c|c|c}
\hline & Ebank1 & Ebank2 & Ebank3 & Ebank4 & Ebank5 & Ebank6 \\
\hline Steps & no installation & 13 & 14 & 19 & 15 & 23 \\
\hline Codes & no installation & 3 & 4 & 4 & 3 & 4 \\
\hline Concepts & no installation & 8 & 8 & 14 & 12 & 8 \\
\hline
\end{tabular}

Table 3. The complexity of the installation process. 


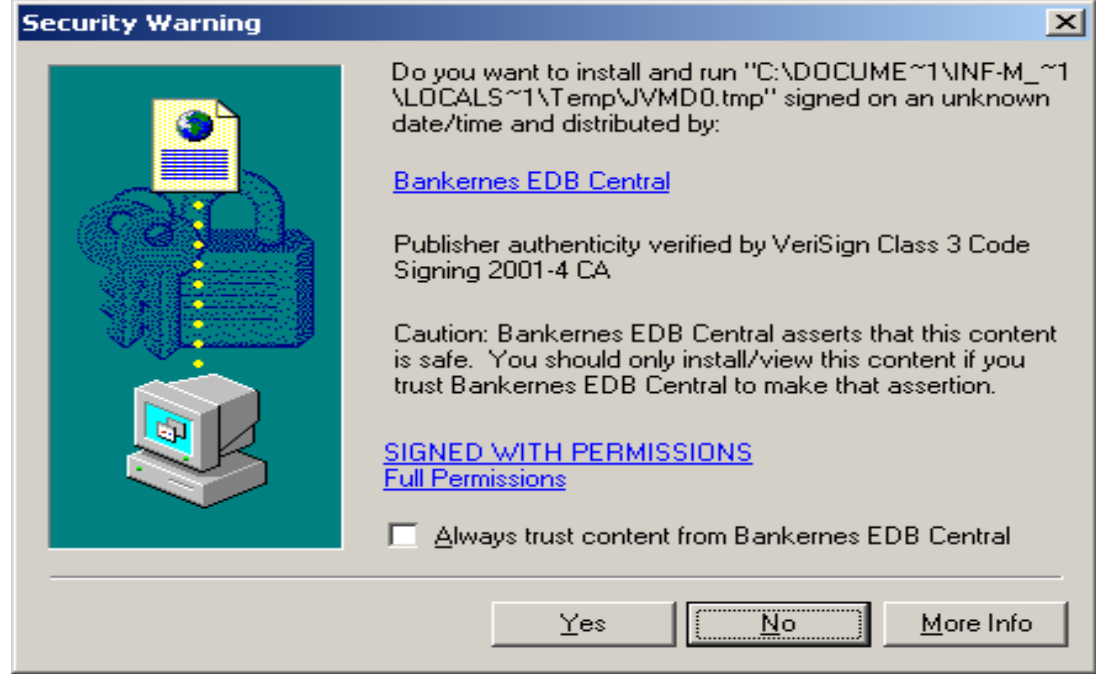

Figure 1. A browser message that pops up during installation of two the PKI-based e-banks. The message is triggered by download of code from the e-bank.

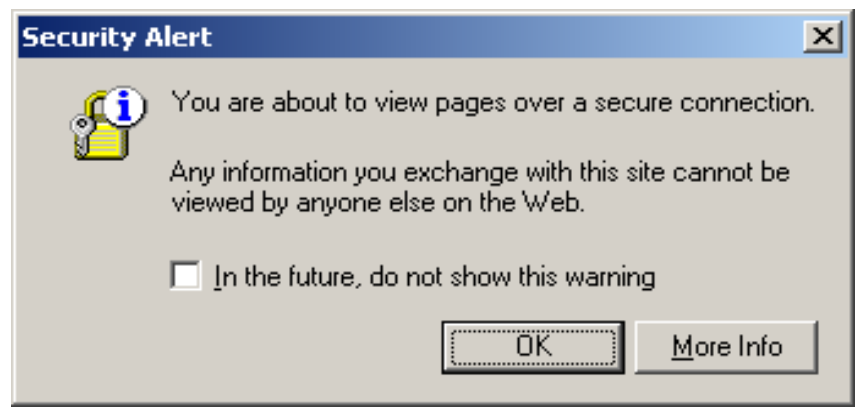

Figure 2. A browser message that pops up during log on to all e-banks. The message is triggered by the initiation of a secure socket connection. 
The table gives the number of steps users must go through, the number of different security-related codes users must key in, and the number of security concepts users are presented with during installation (includes concepts that appear in browser messages).

\section{The browser messages}

In all six e-banking systems, information from the banks is interspersed with security messages from the browser during installation (if any) and during logon and logoff. Figure 1 shows a browser message of a type that appears during installation of two e-banks; one of these provides written material to instruct users what to do, while the other e-bank does not prepare the user. Figure 2 shows a type of browser message that appears at log on to all the e-banks, though only one of them attempts to prepare the user. Thus in many cases users are unprepared for the browser messages, and may experience them as an indication that there is something wrong. In fact the messages are part of normal operation, where sound browser design prescribes that the user is asked about code download.

Most users, even if they recognize that the browser messages are part of normal procedures, will be unable to understand and assess the security implications of the messages. The security warning in Figure 1 asks for the user's permission to install and run a piece of software on the user's computer. There are, however, multiple problems, including: (1) Users cannot be sure that the piece of software is in fact distributed by the entity that claims to be the distributor. (2) Hardly any users will know what an authenticity verification by "VeriSign Class 3 Code Signing 2001-4 CA" is, or whether it is trustworthy. (3) The security warning violates established usability principles such as speak the user's language (Nielsen \& Molich, 1990) and support internal locus of control (Shneiderman, 1998).

The reason that the e-banks, in most cases, do not attempt to explain the browser messages may be that users have different browsers, versions, and configurations, so that the banks are unable to predict what messages are presented to the individual user by the browser. The e-bank that attempts to prepare the user for the message shown in Figure 2 simply writes "If there is a 'Security message', click OK". Indeed, the user has no alternative but to comply at each and every step, unless the user is prepared to discard the e-banking systems altogether. This may overshadow the user's legitimate security concerns.

\section{The user's awareness of and ability to successfully perform the security subtasks during installation}

The security subtasks of installation are password definition and signature-file management. The user is prompted to define a password and a path (possibly a suggested default) for the signature file during installation.

When prompted for password definition, the user is about halfway through installation and has been presented with one or more browser messages. Unfortunately many users at this stage may have already refrained from understanding the relevant security issues, started to disregard his or her security concerns, and may proceed simply by following instructions and accepting defaults. Keeping in mind that the user-defined password serves to protect the private key from disclosure, the dialogues for creating and changing passwords display severe shortcomings:

Not enforcing strong passwords. Four of the five PKI-based banks do not enforce creation of strong passwords. In fact, three banks accept passwords that consist of multiple instances of the same character (e.g., 'aaaaaaaa') and one bank accepts passwords consisting of a digit followed by a sequence of instances of the same letter. Ebank3 enforces several strongpassword rules. The only rule consistently enforced by the banks is that passwords must be at least eight characters long. 
Insufficient information about strong passwords. Three of the banks provide no information about what constitutes a strong password. These banks leave it entirely to users to be aware of possible threats, to realize the consequences of these threats in relation to password creation, and to think of ways of creating strong but memorizable passwords. The two remaining PKIbased banks warn against using real words and personal information (e.g., phone number). One of these banks also suggests how to create strong passwords, including several examples. The examples make the suggestions easier to understand but are at the same time a security weakness because experience shows that a disproportionate number of users will choose the examples, which are visible to attackers, as their passwords.

Preventing strong passwords. Three of the banks restrict passwords to letters and digits, and Ebank3 uses case-insensitive passwords. This significantly reduces the number of possible passwords, and increase their vulnerability to dictionary attacks. (Obviously, simplifications such as case-insensitivity make correct typing of passwords easier.)

Management of the signature file may involve defining a non-standard file path, or storing the file on an external medium instead of on the computer. Such measures may reduce the risk of theft of the signature file, but are only partly supported. An obvious and easily predictable task is a change of password in the situation where the user has made a backup copy of the signature file. Only two of the five PKI-based e-banks tell the user, during the dialogue for changing passwords, that a new backup copy should be made (the reason is that the old backup copy of the signature file still needs the old password for decryption of the private key). There are many other further issues in signaturefile management, such as transferring it to a new computer, that are not explained to the user at all.

\section{THREE APPROACHES TO USABLE SECURITY}

Several of the weaknesses of the e-banking systems discussed above could, in our judgement, be overcome without fundamental changes in the design of the systems. Suggested changes include explaining and enforcing strong passwords, using the same code for temporary and permanent identification, and using a consistent terminology throughout the user interface. However the confusing browser messages (found in all six e-banks) and the complexity of the installation task (the five PKI-based e-banks) would remain, suggesting that more fundamental changes should at least be considered.

Three approaches to the design of usable secure systems are illustrated in Table 4. Automation merely requires that users know of security in the sense that they are aware of the existence and importance of security. However, automation alone is not enough because security is critically dependent on steps and precautions taken by users. Instruction focuses on providing users with the how-to knowledge they need to complete security tasks in a step-by-step manner, without necessarily knowing why steps are taken or how individual steps are related to each other. Understanding attempts to provide users with knowledge of security principles and relies on users to understand these principles sufficiently well to be able to take competent action in an unspecified range of situations. It may be useful to discuss usable security from the point of view of how various designs combine these three approaches. The six e-banking systems are biased toward instruction, and one may consider if the usability of their security facilities could be improved via greater emphasis on automation and/or understanding.

\section{Instruction}

The advantage of instruction, the main approach of all six e-banks, is that the complexity inflicted upon users is reduced by instructing them what to do at each step of the process. This can be viewed as a standard approach, and as already noted, Whitten and Tygar's definition of usable security 
appears to assume that there is a given set of security tasks which the user can be made aware of and instructed about how to perform.

However, instructions can only cover tasks predicted by system designers; they are useless when users are faced with novel tasks not covered by the instructions. This is a critical limitation because unpredicted tasks tend to be quite frequent in complex domains (Rasmussen et al., 1994). The most glaring example of the limitations of instruction in relation to the e-banking systems is that part of the functionality and user interaction is outside the control of the e-banking systems because it is performed by the browser. Users may have different browsers, different versions of the same browser, or their browsers may be set up differently. This defeats thorough instruction because neither the sequence of steps a user will go through nor their exact contents is known to the ebanking system. Relying on instruction in such a situation involves a risk of confusing and frustrating users due to mismatches between the instructions and the actual system behaviour. Since instruction lends itself to a dialogue where the system is in command and the user is assigned a residual role of filling in missing pieces of information, instruction also entails a risk of compromising security by confronting users with issues not covered by the instructions but nevertheless issues users need to make informed decisions about.

\section{Automation}

Automation may be considered a possible way of simplifying the user interfaces of the e-banking systems, for example in order to minimize the number of steps users must go through (see Tables 2 and 3). One should note that the banks have already automated complex parts of the processes involved in secure use of the e-banks. For example, the PKI-based e-banks partially automate key generation and digital signing of a payment transaction. Of the seven steps listed by Schultz et al. (2001) as typical for digital signing, four are automated. This is due to the integration of the digital signature into the e-banking system.

A candidate for further automation would be a 'Remember password' function, for the password that protects the digital signature. Along with Schultz' et al. we find that this would introduce an unacceptable risk, because the legitimate user may leave the computer unattended. A better way to reduce the number of times the password must be typed is to allow for packaging of transactions, similarly to collecting multiple items in a shopping basket prior to purchase on an e-commerce site. Another candidate for automation is the browser-based dialogs, because the browser messages are confusing and the user always must type the same answer, eg., "Yes" in the dialog shown in Figure 1. It might be possible to automate this step if the banks provide the user with a specialized user interface, for example a preconfigured browser dedicated to e-banking. However, this approach appears as a step backwards to PC-banking, the previous generation of electronic banking, in that it eliminates the advantage for users (in terms of convenience) and banks (in terms of maintenance costs) of reusing existing and well-known web-components.

A third candidate for automation is the selection of a pathname for the signature file. However, removing the option of selecting a non-default path weakens security, because it makes it easier for hostile programs, transferred by a remote attacker to the user's computer, to obtain a copy of the file. All in all, further automation does not seem to be a viable road given the web-based architecture and the requirement that authentication is based, in part, on a secret the user knows, as required - rightly, we think - in the Danish industry code for e-banking.

\section{Understanding}

A major obstacle to providing the user with an understanding of security aspects of e-banking is the unforgiving nature of security breaches, i.e. the barn-door property mentioned in the Usable Security section. Widely used usability heuristics such as 'permit easy reversal of actions' (Shneiderman, 
1998) carry with them the underlying assumption that designs should encourage exploration or, at least, allow for a trial-and-error approach to learning how to use systems. However, for e-banking and other security-sensitive systems, a trial-and-error approach is generally not acceptable because a security breach caused by an error may be exploited by an attacker before the error is revoked by the user. Once a secret has been left accidentally unprotected, even for a short time, there is no way to be sure that it has not already been read by an attacker. This adds to the demands involved in learning to use these systems and sets this learning process apart from how most users learn to use text processors, spreadsheets, and similar systems.

Several questionnaire studies have attempted to identify the factors that influence the adoption of ebanking systems (e.g., Liao \& Cheung, 2002; Sohail \& Shanmugham, 2003; Tan \& Teo, 2000). These studies unanimously find that security is important to users. A significant portion of the security understanding users must have to perform their e-banking transactions in a safe manner is not specific to e-banking but, rather, part of a general requirement for behaving safely and competently in a digitally networked world (e.g., Claessens et al., 2002; Schneier, 2000; Whitman, 2003). However, most users may not be motivated to learn about security and may instead tend to experience it as an obstacle that complicates and slows down their business with their e-bank. Users may be tempted to put off learning about security, since they generally do not sit down at their computers wanting to scrutinize the consequences of suggested security defaults. The security skills and active user involvement required to understand security issues may be more than most users are prepared to deliver.

\begin{tabular}{|l|l|l|l|}
\hline Approach to security & Automation & Instruction & Understanding \\
\hline User's frame of mind & Trust & Procedural compliance & Active involvement \\
\hline Task coverage & Formalized tasks only & Predicted tasks only & Predicted and unpredicted tasks \\
\hline Type of user knowledge & Awareness knowledge & How-to-knowledge & Principles knowledge \\
\hline
\end{tabular}

Table 4. Three approaches to usable security. From a pragmatic point of view, instruction appears the main approach, but to handle complex security issues aspects of automation and understanding must be incorporated.

\section{CONCLUSION}

Attaining both security and ease of use in e-banking systems is difficult because security is not a system feature that can be provided automatically while users focus on their primary goal of accomplishing their business with their bank. In the six surveyed e-banking systems, security and ease of use are traded against each other at three levels:

The individual-features level. Passwords provide a prominent example of a feature that involves a trade-off between security and ease of use. Four of the e-banking systems do not enforce strong passwords. Further, three of the systems have drastically restricted the set of characters that can appear in passwords and thereby prevented strong passwords. Some of these restrictions - for example case insensitivity - make passwords easier to use by avoiding frequent usability problems.

The system-architecture level. Five of the e-banking systems are Public Key Infrastructure solutions and the last e-banking system makes use of one-time codes drawn from a physical card. Both types of security solutions entail considerable complexity within three areas: the number of steps users must go through to complete transactions, the number of security concepts with which users are presented, and the number of security-related codes users must key in during system installation. By relying on the user's browser as their basic platform, the e-banking systems get access to a standard set of well-tested communication and security 
facilities. However, in making use of these facilities the e-banking systems give up some control over how security issues are negotiated with users, and unless the user is prepared to discard the e-banking system there is no alternative to granting the browser the privileges it requests and, hence, no reason to scrutinize even legitimate security concerns.

The basic-assumptions level. The six e-banking systems share the assumption that information provided to users should be step-by-step instructions stipulating how to perform specified tasks. The instructions enable users to perform the specified tasks without concerning themselves with the underlying security issues but at the same time leave users without the understanding required to act competently in situations not covered by the instructions. This is a critical trade-off because, on the one hand, e-banking users cannot generally be expected to be motivated to learn about security but, on the other hand, they need to understand it well enough to avoid unsafe actions. The bias toward instruction gives priority to ease of use in predicted situations at the expense of making the systems more difficult to use safely in an unknown range of unpredicted situations.

We conclude that most users are only able to complete installation of the e-banking systems by painstakingly following instructions, accepting defaults, and refraining from any real understanding of the involved security issues. However, the high penetration of e-banking systems in Denmark suggests that many people are prepared to trust e-banking systems in the absence of compelling, publicly known evidence about security breaches leading to financial loss by e-banking customers.

\section{REFERENCES}

Adams, A., Sasse, M.A., and Lunt, P. (1997) "Making passwords secure and usable" in People and Computers XII: Proceedings of HCI'97, Springer, Berlin, pp. 1-19.

Beyer, H., and Holtzblatt, K. (1998) Contextual Design: Defining Customer-Centered Systems, Morgan Kaufmann, San Francisco, CA.

Claessens, J., Dem, V., Cock, D.D., Preneel, B., and Vandewalle, J. (2002) On the security of today's electronic banking systems, Computers \& Security, 21(3), 257-269.

Dewan, R., and Seidmann, A. (eds.) (2001) Current issues in e-banking (Special section), Communications of the ACM, 44(6), 31-57.

Dourish, P., and Redmiles, D. (2002) "An approach to usable security based on event monitoring and visualization" in Proceedings of the 2002 Workshop on New Security Paradigms, ACM Press, New York, pp. 75-81.

Hertzum, M., Juul, N.C., Jørgensen, N., and Nørgaard, M. (2004) Usable Security and E-Banking: Ease of Use vis-à-vis Security. Technical Report. URL: http://www.ruc.dk/ nielsj/research/papers/ebanking-tr.pdf.

IDC (2003) Total IT security market - including software, hardware, and services - to reach $\$ 45$ billion by 2006, according to IDC (press release), IDC, Framingham, MA. Available at: www.idc.com/getdoc.jhtml?containerId=pr2003_01_28_085549. Consulted: September 19, 2003.

ISO 9241-11 (1998) Ergonomic Requirements for Office Work with Visual Display Terminals (VDTs) - Part 11: Guidance on Usability, International Organization for Standardization, Geneva.

ISO/IEC 9126-1 (2001) Software Engineering - Product Quality - Part 1: Quality Model, International Organization for Standardization, Geneva.

ITU (1991) Security Architecture for Open Systems Interconnection for CCITT Applications. Recommendation X.800, International Telecommunication Union, Geneva.

Jih, W.-J., Wong, S.-Y., and Chang, T.-B. (2005) Effects of perceived risks on adoption of Internet banking services: an empirical investigation in Taiwan, International Journal of E-Business Research, 1(1), 70-88.

AJIS Special Issue

December 2004 
Klein, D.V. (1990) "'Foiling the cracker': a survey of, and improvements to, password security" in Proceedings of the Second USENIX Security Workshop, USENIX, Berkeley, CA, pp. 514.

Liao, Z., and Cheung, T. (2002) Internet-based e-banking and consumer attitudes: an empirical study, Information \& Management, 39(4), 283-295.

Morris, R., and Thompson, K. (1979) Password security: a case history, Communications of the ACM, 22(11), 594-597.

Nielsen, J., and Molich; R. (1990) "Heuristic evaluation of user interfaces" in Proceedings of the ACM CHI '90 Conference on Human Factors in Computing systems, ACM Press, New York, pp. 249-256.

OECD (2001) Electronic Finance: Economics and Institutional Factors, Occasional Papers No. 2, Financial Affairs Division, Organisation for Economic Co-operation and Development, Paris, France.

Pinkas, B., and Sander, T. (2002) "Securing passwords against dictionary attacks" in Proceedings of the 9th ACM Conference on Computer and Communications Security, ACM Press, New York, pp. 161-170.

Rasmussen, J., Pejtersen, A.M., and Goodstein, L.P. (1994) Cognitive Systems Engineering, Wiley, New York.

Schneier, B. (2000) Secrets and Lies: Digital Security in a Networked World, Wiley, New York.

Schultz, E.E., Proctor, R.W., Lien, M.-C., and Salvendy, G. (2001) Usability and security: an appraisal of usability issues in information security methods, Computers \& Security, 20(7), 620-634.

Shneiderman, B. (1998) Designing the User Interface: Strategies for Effective HumanComputer Interaction, Third Edition, Addison Wesley, Reading, MA.

Smetters, D.K., and Grinter, R.E. (2002) "Moving from the design of usable security technologies to the design of useful secure applications" in Proceedings of the 2002 Workshop on New Security Paradigms, ACM Press, New York, pp. 82-89.

Sohail, M.S., and Shanmugham, B. (2003) E-banking and customer preferences in Malaysia: an empirical investigation, Information Sciences, 150(3\&4), 207-217.

Statistics Denmark (2004). Information Society Denmark. ICT Status 2004. URL: http://www.dst.dk/infosamfundet2004.aspx.

Tan, M., and Teo, T.S.H. (2000) Factors influencing the adoption of internet banking, Journal of the Association for Information Systems, 1, Article 5. Available at: http://jais.isworld.org/articles/ default.asp?vol=1\&art=5. Consulted: December 5, 2003.

Whitman, M.E. (2003) Enemy at the gate: threats to information security, Communications of the ACM, 46(8), 91-95.

Whitten, A., and Tygar, J.D. (1999) "Why Johnny can't encrypt: a usability evaluation of PGP 5.0" in Proceedings of the 8th USENIX Security Symposium, USENIX, Berkeley, CA.

\section{ACKNOWLEDGEMENTS}

Thanks to Niels Christian Juul for contributions to this study. The study was supported, in part, by the Development Centre for Electronic Business, Copenhagen Business School, and the IT University of Copenhagen.

\section{COPYRIGHT}

Morten Hertzum, Niels Christian Juul, Niels Jørgensen, Mie Nørgaard (C 2004. The authors assign to OZCHI and educational and non-profit institutions a non-exclusive licence to use this document for personal use and in courses of instruction provided that the article is used in full and this copyright AJIS Special Issue

December 2004 
statement is reproduced. The authors also grant a non-exclusive licence to OZCHI to publish this document in full in the Conference Papers and Proceedings. Those documents may be published on the World Wide Web, CD-ROM, in printed form, and on mirror sites on the World Wide Web. Any other usage is prohibited without the express permission of the authors. 